\title{
NOUVELle
}

\section{Le double visage \\ de la Sestrine 2 dans \\ les processus pathologiques liés au vieillissement}

Fanny Lebourg ${ }^{1}$, Sloane Paulcan ${ }^{1}$, Michel Lepoivre ${ }^{2}$

\author{
${ }^{1}$ Ml Biologie Santé, Université Paris-Saclay, 91405 Orsay, \\ France ; \\ ${ }^{2}$ Laboratoire bioénergétique membranaire et stress, UMR \\ CNRS 9198, I2BC (institut de biologie intégrative de la cellule), \\ université Paris-Saclay, bâtiment 430, Orsay, 91405, France. \\ fanny.lebourg@u-psud.fr \\ sloane.paulcan@u-psud.fr \\ michel.lepoivre@u-psud.fr
}

> Le vieillissement physiologique est caractérisé par de multiples modifications moléculaires, cellulaires et fonctionnelles dans la genèse desquelles l'accumulation des espèces réactives dérivées de l'oxygène (ROS) joue un rôle important. Les ROS, produits en particulier par les mitochondries, peuvent induire des dommages oxydants délétères, tels que la peroxydation lipidique. La voie anabolique mTOR (mammalian target of rapamycin) est aussi impliquée dans le vieillissement cellulaire. Dans plusieurs modèles animaux, une inhibition de mTOR accroît la longévité [1], par induction de la mitophagie et du catabolisme des protéines ou lipides dysfonctionnels.

Les sestrines (Sesn) font partie d'une grande famille de protéines très conservées chez les métazoaires, dont trois membres sont présents chez les mammifères (Sesn 1, 2 et 3) [2]. Elles exercent notamment des fonctions de protection antioxydante et de contrôle du métabolisme via l'inhibition de la voie mTOR. L'expression des Sesnl et 2 est induite par la protéine antitumorale p53 [3]. La Sesn2, qui est aussi régulée en condition d'hypoxie par le facteur de transcription HIF-1 (hypoxia-inducible factor 1), permettrait de protéger de nombreux organes contre diverses agressions liées au stress oxydant et au vieillissement [4].

La structure moléculaire des Sesn était inconnue jusqu'à récemment. Sa réso-

Cette Nouvelle fait partie d'une série de 15 Nouvelles rédigées par les étudiants du Master «Biologie Santé » de l'université Paris-Saclay, qui paraîtront dans les numéros $6-7,8-9,10$ et 11 (2016) de médecine/sciences. lution permet de comprendre comment une unique protéine peut porter deux fonctions apparemment très distinctes, d'une part la détoxification des ROS, et, d'autre part, l'inhibition de la voie mTOR. Cette dualité amène à supposer qu'une régulation simultanée de ces deux activités serait utile dans certaines situations, notamment pour atténuer les processus dégénératifs liés au vieillissement.

\section{Résolution de la structure}

de la hSesn2 : un pas en avant vers la compréhension d'une double régulation métabolique

La structure de la Sesn2 humaine (hSesn2) - révélée par l'équipe de U.S. Cho en 2015 - est monomérique, pseudo-symétrique et composée de trois domaines [5]. Deux d'entre eux, Sesn-A et $C$, sont très similaires et adoptent une forme globulaire composée d'hélices $\alpha$ (Figure 1). Ils sont reliés par le domaine Sesn-B, un motif hélice-boucle-hélice dont le rôle reste inconnu.

Une analyse d'homologie de séquence et de structure a révélé que chacun des domaines Sesn-A et $C$ présente une grande homologie avec YP_296737.1, une protéine bactérienne dimérique de Ralstonia eutropha et hypothétique hydroperoxydase d'alkyles'. Ceci suggère qu'un monomère de YP_296737.1 pour-

${ }^{1}$ Les hydroperoxydes d'alkyles, de formule ROOH, résultent de I'oxydation par des ROS (ex : $\mathrm{H}_{2} \mathrm{O}_{2}$ ) d'acides gras insaturés, de glucides ou de protéines. La peroxydation des lipides altère les propriétés des membranes biologiques. Elle contribue aussi au développement des pathologies cardiovasculaires. Les hydroperoxydases d'alkyle catalysent la réduction des hydroperoxydes $\mathrm{ROOH}$ et permettent ainsi l'élimination de ces composés délétères. rait avoir été dupliqué dans hSesn2. Des divergences existent néanmoins entre les deux domaines: Sesn-A porte un motif hélice-boucle-hélice, spécifique des hydroperoxydases d'alkyles telles qu'AhpD de Mycobacterium tuberculosis, et retrouvé dans YP_296737.1, alors que Sesn-C ne porte qu'un motif héliceboucle. La protéine hSesn2 présente ainsi deux domaines symétriques, mais structurellement différents.

Afin de déterminer l'implication de Sesn-A dans l'activité détoxifiante de la hSesn2 vis-à-vis des ROS, des tests d'activité ont été réalisés en utilisant différents substrats. Ils démontrent que la hSesn2 est capable de lier et de réduire l'hydroperoxyde de cumène, un hydroperoxyde hydrophobe de synthèse. Le peroxyde d'hydrogène $\left(\mathrm{H}_{2} \mathrm{O}_{2}\right)$ n'est pas dégradé par la hSesn2, mais bien par l'AhpD. Les auteurs de cette étude expliquent cette différence par l'environnement hydrophobe de la cystéine catalytique de hSesn2, identifiée comme la Cys125 du domaine Sesn-A. En effet, la mutation C125S diminue de $95 \%$ l'activité hydroperoxydase d'alkyles de la sestrine. C125 est oxydée en acide sulfénique au cours de la réduction du substrat, et cette réaction a lieu également dans des cellules en culture traitées par l'hydroperoxyde de cumène. L'acide sulfénique résultant (C125-SOH) est par la suite régénéré en cystéine par un réducteur dont l'identité est inconnue. Les auteurs proposent que les substrats physiologiques de la Sesn2 soient des lipides peroxydés, qui sont détectés dans de multiples pathologies liées au stress oxydant. Si de nombreuses 


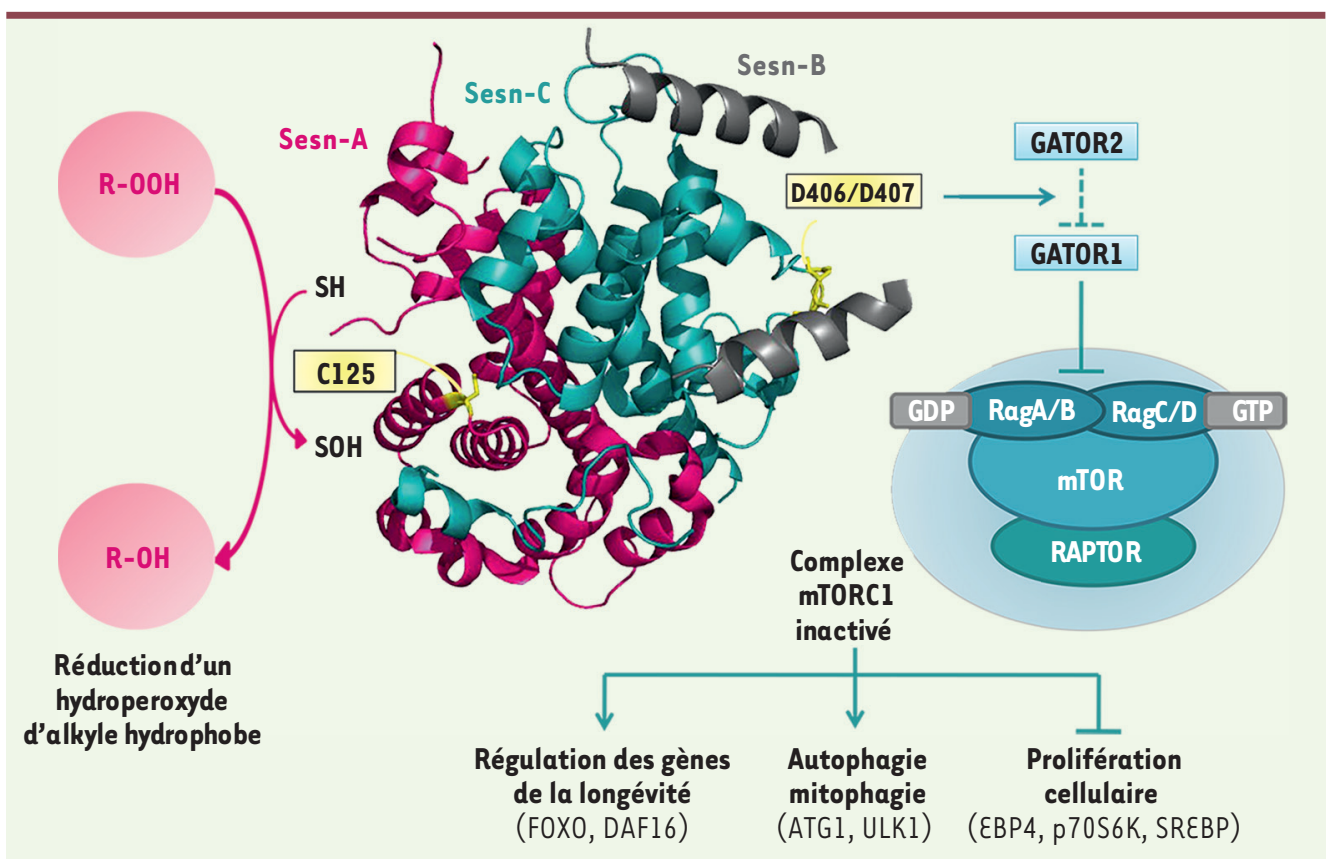

Figure 1. Représentation schématique des deux activités de la hSesn2. La protéine est composée de trois domaines, Sesn-A (magenta), Sesn-B (gris) et Sesn-C (turquoise). Sesn-A et Sesn-C sont similaires mais distincts par leurs fonctions respectives. Le domaine Sesn-A permet la réduction de peroxydes d'alkyles hydrophobes (vraisemblablement des lipides peroxydés) via la cystéine catalytique C125, transitoirement oxydée en acide sulfénique (R-SOH). Le domaine Sesn-C est, lui, responsable de l'inhibition

de mTORC1 (mTOR complex 1) par liaison directe au régulateur GATOR2 (GAP activity towards Rags 2). Ceci stimule l'autophagie et inhibe des processus anaboliques consommateurs d'ATP. Ce double-visage de la hSesn2 contre l'accumulation de ROS et l'activation de la voie mTOR permet d'atténuer des processus métaboliques impliqués dans le vieillissement. FOXO : forkhead box class 0; DAF16: homologue de la famille F0X0; ATG1 : autophagye related 1 ; ULK1 : Unc-51 like autophagy activating kinase 1 ; EBP4 : Emopamil binding protein 4 ; p70S6K : p70 ribosomal S6 kinase ; SREBP : sterol regulatory element-binding protein; Raptor : regulatory-associated protein of mTOR.

études confirment le rôle antioxydant de la Sesn2, son activité de réductase de l'acide sulfinique des peroxyrédoxines suroxydées (Prx-S02H) proposée initialement, est aujourd'hui contestée [6]. On note que la Sesn2 ne présente aucune similitude structurale avec la sulfirédoxine, la seule réductase des Prx-SO2H identifiée à ce jour.

Selon les auteurs, le domaine Sesn-C semble avoir évolué différemment de Sesn-A au sein de hSesn2. En effet, Sesn-C a perdu le motif hélice-bouclehélice qui constitue la signature structurale des homologues YP_296737.1/ AhpD/Sesn-A. II est remplacé par un motif hélice-boucle, responsable de l'inhibition de mTORCl (mTOR complex 1) par Sesn2. Les résidus impliqués dans cette fonction inhibitrice ont été identifiés par mutagenèse dirigée. II s'agit des deux Asp406 et 407 nommés motif DD (Figure 1). Ces deux résidus appartiennent au motif hélice-boucle, suggérant que Sesn-C aurait acquis sa fonction régulatrice de $\mathrm{mTORCl}$ en modifiant le motif hélice-boucle-hélice. Ce motif DD est crucial pour l'interaction de la hSesn2 avec GATOR2 (GAP activity towards Rags 2), permettant la libération de GATORl. Celui-ci inhibe l'activité des Rag-GTPases (recombination activating genes GTPases) et ainsi le complexe mTORCl. L'inhibition de mTORCl aboutit à la transcription de gènes impliqués dans la longévité, l'autophagie et l'inhibition de la prolifération cellulaire.

Grâce à ses deux domaines indépendants Sesn-A et C, la hSesn2 a donc bien une double activité, réductrice des ROS et inhibitrice de la voie mTOR, impliquée dans la régulation de l'homéostasie métabolique. La hSesn2 montre ainsi un caractère unique avec une structure similaire à tête de Janus, dieu grec aux deux visages opposés.

\section{hSesn2 : une protéine régulatrice du} vieillissement encore méconnue La première structure cristallographique de la Sesn2 permet de comprendre la dualité fonctionnelle de cette protéine. Les deux activités - réductrice des ROS et inhibitrice de la voie mTOR - s'opposent au développement de processus pathologiques et de troubles métaboliques qui accompagnent en particulier le vieillissement et l'obésité (accumulation de lipides et de plaques d'athérome, dysfonction mitochondriale, agrégation protéique, diabète, etc.). L'énergie cellulaire est ainsi adaptée au profit d'une réponse au stress, en éliminant les mitochondries dysfonctionnelles ou des protéines agrégées par exemple. Cependant, la raison de la colocalisation de deux domaines distincts portant des fonctions métaboliques très différentes dans une même structure reste incomprise. Nous supposons que l'expression d'un seul polypeptide portant ces deux fonctions permet de lutter simultanément contre deux processus qui, chacun, concourt aux mêmes dérèglements pathophysiologiques, notamment des processus dégénératifs liés au vieillissement. 
De précédentes études ont montré que la Sesn2 était aussi capable de potentialiser l'action de Nrf2 (nuclear factor [erythroid-derived 2]-like 2), un facteur de transcription gouvernant une réponse génique antioxydante. Par la formation d'un complexe Sesn2-p62-Rbxl (RINGbox protein 1 , une ubiquitine ligase), la protéine Keapl (Kelch-like $\varepsilon C H$-associated protein 1 ) peut être dégradée par autophagie, levant son inhibition sur Nrf2. Cette action pourrait compléter l'activité détoxifiante antiperoxyde du domaine Sesn-A de la Sesn2 [7]. Puisque la protéine $\mathrm{p} 62$ peut appartenir au complexe mTORCl, elle pourrait être un lien de coordination entre les deux activités de la Sesn2 [8].
Par son expression ubiquitaire et la pluralité de ses actions, la Sesn2 apparaît comme un effecteur d'un grand intérêt dans l'homéostasie métabolique et la prévention du vieillissement. D'autres études sont encore nécessaires pour mieux comprendre toutes les facettes de cette protéine. $\diamond$

The two-faced Sestrin2 against

age-related pathologies

\section{LIENS D'INTÉRÊT}

Les auteurs déclarent n'avoir aucun lien d'intérêt concernant les données publiées dans cet article.

\section{RÉFÉRENCES}

1. Harrison DE, Strong R, Sharp ZD, et al. Rapamycin fed late in life extends lifespan in genetically heterogeneous mice. Nature 2009 ; 460 : 392-5.
2. Budanov AV, Lee JH, Karin M. Stressin' sestrins take an aging fight. EMBO Mol Med $2010 ; 2$ : 388-400.

3. Budanov AV, Karin M. p53 target genes sestrinl and sestrin2 connect genotoxic stress and mTOR signaling Cell 2008 ; $134: 451-60$.

4. Budanov AV, Shoshani T, Faerman A, et al. Identification of a novel stress-responsive gene Hi95 involved in regulation of cell viability. Oncogene $2002 ; 21: 6017-31$.

5. Kim H, An S, Ro SH, et al. Janus-faced Sestrin2 controls ROS and mTOR signalling through two separate functional domains. Nat Commun 2015 ; 6 : 10025.

6. Woo H, Bae S, Park S, et al. Sestrin 2 is not a reductase for cysteine sulfinic acid of peroxiredoxins. Antioxid Redox Signal 2009 ; 11 : 739-45.

7. Bae SH, Sung SH, Oh Sy, et al. Sestrins activate Nrf2 by promoting p62-dependent autophagic degradation of Keapl and prevent oxidative liverdamage. Cell Metab $2013 ; 17: 73-84$.

8. Duran A, Amanchy R, Linares JF, et al. p62 is a key regulator of nutrient sensing in the mTORCl pathway. Mol Cell $2011 ; 44: 134-46$.
NOUVELLE

\section{Crystal Wars : la nécroptose contre-attaque}

Diana García Garcíal ${ }^{1}$, Charlotte Duwat ${ }^{1}$, Pierre Bobé ${ }^{2}$

\author{
${ }^{1}$ Ml Biologie Santé, Université \\ Paris-Saclay, 91405 Orsay, France ; \\ ${ }^{2}$ Inserm UMR S 1174, Interactions cellulaires \\ et physiopathologie hépatique, bâtiment \\ 440-443, université Paris Sud, rue des Adèles, \\ 91405 Orsay, France. \\ pierre.bobe@u-psud.fr \\ cduwat@live.fr \\ dianegg25@gmail.com
}

> Une étude récente portant sur des patients atteints de diabète de type II a révélé chez trois d'entre eux une insuffisance rénale aiguë (IRA) secondaire à une néphropathie aiguë à oxalate, caractérisée par la présence de dépôts tubulo-interstitiels d'oxalate, accompagnés d'une réaction inflammatoire [1]. Cette étude montre que les cristallopathies comme la néphropathie aiguë à oxalates - devraient être plus souvent recherchées comme étiologie d'une insuffisance rénale aiguë. Les cristallopathies sont des maladies caractérisées par la présence de cristaux dans

Cette Nouvelle fait partie d'une série de 15 Nouvelles rédigées par les étudiants du Master «Biologie Santé » de l'université Paris-Saclay, qui paraîtront dans les numéros $6-7,8-9,10$ et 11 (2016) de médecine/sciences. différents organes ${ }^{1}$. Ces cristaux sont de tailles et formes variables, composés d'ions ou encore de molécules produites par l'organisme (comme l'urée). La formation des dépôts commence par un processus de nucléation - qui correspond à l'apparition des premiers germes cristallins dans les tissus - suivi par la croissance de ces germes jusqu'à former des cristaux.

De nombreuses recherches ont déjà montré que la nucléation, la formation et

\footnotetext{
${ }^{1}$ On distingue quatre sous-groupes de cristallopathies: (1) certaines sont communes, par exemple goutte par dépôts d'urate de sodium dans les articulations, maladies par dépôts de cristaux de pyrophosphate de calcium (chondrocalcinose articulaire) ou d'hydroxyapatite; (2) certaines se rencontrent dans le cadre de maladies endocrines ou métaboliques, par exemple un hyperparathyroïdisme, une hypothyroïdie, une acromégalie ; (3) des cristallopathies peuvent être associées à une maladie génétique, par exemple une hémochromatose; (4) enfin, il existe des cristallopathies iatrogéniques, secondaires par exemple à des injections intra-articulaires de corticostéroïdes.
}

la présence des cristaux dans les tissus cibles des cristallopathies sont la cause des lésions, de l'inflammation et du remodelage tissulaires. Cependant, il reste encore une part d'inconnu dans ce type de maladie. En effet, il n'a pas encore été déterminé quelle est la part, dans les lésions, de l'inflammation (effet indirect) et des cristaux eux-mêmes (effet direct) et, dans ce dernier cas, s'il s'agit d'un effet purement mécanique ou d'un processus régulé. L'équipe de H.J. Anders de l'université de Munich [2] explore l'hypothèse d'un processus régulé à l'origine des lésions, et permet de mieux comprendre les voies de signalisation impliquées dans ce type de pathologie.

\section{Comment les cristallopathies sont-elles produites?}

Les cristaux sont reconnus par le système immunitaire inné comme des 